\title{
Multifractality at the spin quantum Hall transition
}

\author{
F. Evers ${ }^{1}$ A. Mildenberger, ${ }^{1}$ and A. D. Mirlin ${ }^{1,2, *}$ \\ ${ }^{1}$ Institut für Nanotechnologie, Forschungszentrum Karlsruhe, 76021 Karlsruhe, Germany \\ ${ }^{2}$ Institut für Theorie der Kondensierten Materie, Universität Karlsruhe, 76128 Karlsruhe, Germany
}

(Received 8 November 2002; published 17 January 2003)

\begin{abstract}
Statistical properties of critical wave functions at the spin quantum Hall transition are studied both numerically and analytically (via mapping onto the classical percolation). It is shown that the index $\eta$ characterizing the decay of wave function correlations is equal to $1 / 4$, at variance with the $r^{-1 / 2}$ decay of the diffusion propagator. The multifractality spectra of eigenfunctions and of two-point conductances are found to be close to parabolic, $\Delta_{q} \simeq q(1-q) / 8$ and $X_{q} \simeq q(3-q) / 4$.
\end{abstract}

Disordered two-dimensional electron systems show remarkably rich physics which is governed by quantum interference effects and depends on the symmetry class to which the system belongs. Recently, unconventional symmetry classes, ${ }^{1}$ which can be realized in $d$-wave superconductors, have attracted considerable interest. Particular attention was paid to class $\mathrm{C}$ (broken time-reversal invariance), where a transition between localized phases with quantized values of the spin Hall conductance takes place. ${ }^{2-4}$ A network model describing this spin quantum Hall ( $\mathrm{SQH}$ ) transition was constructed in Ref. 2, and critical exponents for the scaling of the localization length were determined numerically. In Ref. 3 a mapping onto a supersymmetric spin chain was performed, providing an alternative method for the numerical study of the critical behavior. Remarkably, some exact analytical results for this model have been obtained by mapping onto the classical percolation problem. ${ }^{4,5}$ In particular, it was found that the density of states (DOS) is critical and scales as $\rho(\epsilon) \sim \epsilon^{1 / 7}$, while the diffusion propagator $\Pi\left(\mathbf{r}, \mathbf{r}^{\prime}\right)$ $=\left\langle G_{R}\left(\mathbf{r}, \mathbf{r}^{\prime}\right) G_{A}\left(\mathbf{r}^{\prime}, \mathbf{r}\right)\right\rangle$ and the average two-point conductance $\left\langle g\left(\mathbf{r}, \mathbf{r}^{\prime}\right)\right\rangle$ fall off as $\left|\mathbf{r}-\mathbf{r}^{\prime}\right|^{-1 / 2}$ at criticality.

Multifractality of wave functions $\psi(\mathbf{r})$ is known to be a hallmark of the localization transition. It has been extensively studied in the context of conventional Anderson and quantum Hall $(\mathrm{QH})$ transitions with noncritical DOS (see, e.g., Ref. 6 and references therein), and we remind the reader of some basic results. Multifractality is characterized by a set of exponents $\tau_{q} \equiv d(q-1)+\Delta_{q}$ ( $d$ is the spatial dimensionality) describing the scaling of the moments of $\left|\psi^{2}(\mathbf{r})\right|$ with the system size, $\left\langle|\psi(\mathbf{r})|^{2 q}\right\rangle \propto L^{-d-\tau_{q}}$. Anomalous dimensions $\Delta_{q}$ distinguish a critical point from the metallic phase and determine the scale dependence of wave function correlations. Among them, $\Delta_{2} \equiv-\eta$ plays the most prominent role, governing the spatial correlations of the "intensity" $|\psi|^{2}$ :

$$
L^{2 d}\left\langle\left|\psi^{2}(\mathbf{r}) \psi^{2}\left(\mathbf{r}^{\prime}\right)\right|\right\rangle \sim\left(\left|\mathbf{r}-\mathbf{r}^{\prime}\right| / L\right)^{-\eta} .
$$

Correlations of two different (but close in energy) eigenfunctions and the diffusion propagator possess the same scaling properties,

$$
\begin{gathered}
L^{2 d}\left\langle\left|\psi_{i}^{2}(\mathbf{r}) \psi_{j}^{2}\left(\mathbf{r}^{\prime}\right)\right|\right\rangle, \quad L^{2 d}\left\langle\psi_{i}(\mathbf{r}) \psi_{j}^{*}(\mathbf{r}) \psi_{i}^{*}\left(\mathbf{r}^{\prime}\right) \psi_{j}\left(\mathbf{r}^{\prime}\right)\right\rangle, \\
\rho^{-2} \Pi\left(\mathbf{r}, \mathbf{r}^{\prime} ; \omega\right) \sim\left(\left|\mathbf{r}-\mathbf{r}^{\prime}\right| / L_{\omega}\right)^{-\eta},
\end{gathered}
$$

where $\omega=\epsilon_{i}-\epsilon_{j}, \quad L_{\omega} \sim(\rho \omega)^{-1 / d}$, and $\left|\mathbf{r}-\mathbf{r}^{\prime}\right|<L_{\omega}$. The moments of the two-point conductance show a power-law scaling as well, ${ }^{7,8}\left\langle g^{q}\left(\mathbf{r}, \mathbf{r}^{\prime}\right)\right\rangle \propto\left|\mathbf{r}-\mathbf{r}^{\prime}\right|^{-X_{q}}$, with another set of exponents $X_{q}$, which are related to $\Delta_{q}$ (Refs. 9 and 10):

$$
X_{q}=\left\{\begin{array}{l}
\Delta_{q}+\Delta_{1-q}, \quad q<1 / 2 \\
2 \Delta_{1 / 2}, \quad q>1 / 2 .
\end{array}\right.
$$

In two dimensions the multifractal spectra $\Delta_{q}$ and $X_{q}$ play a key role in the identification of the conformal field theory of the critical point, which led to growing interest in the eigenfunction statistics at the $\mathrm{QH}$ transition. ${ }^{7-11}$

In this paper, we study, by combining numerical and analytical means, the statistical properties of wave functions at the SQH critical point. Our main aim is to calculate exponents governing the scaling of $\left\langle|\psi|^{2 q}\right\rangle,\left\langle g^{q}\right\rangle$, and $\Pi$, and to understand relations between them for the transition with critical DOS. The Hamiltonian for class $\mathrm{C}$ satisfies the symmetry $H^{*}=-\sigma_{y} H \sigma_{y}$ (with $\sigma_{y}$ the Pauli matrix in the particle-hole space) and has a block structure

$$
H=\left(\begin{array}{ll}
h & \Delta \\
\Delta^{*} & -h^{T}
\end{array}\right), \quad h=h^{\dagger}, \quad \Delta=\Delta^{T} .
$$

For computer simulations we used the SU(2) network model. ${ }^{2,5}$ The dynamics of the wave function defined on edges of the network is governed by a unitary evolution operator $\mathcal{U}=e^{-i H}$. At each node of the network the scattering from two incoming into two outgoing links is described by a matrix $S$, with $S_{11}=S_{12}=S_{22}=-S_{21}=1 / \sqrt{2}$. Each realization of the network is characterized by a set of random 2 $\times 2$ spin matrices $U_{e}$ associated with all edges $e$ of the network. In view of Eq. (4), $\mathcal{U}$ satisfies the symmetry $\mathcal{U}$ $=\sigma_{y} \mathcal{U}^{*} \sigma_{y}$, implying that $U_{e} \in \mathrm{SU}(2)$. Diagonalizing $\mathcal{U}$ for a square network of the size $L \times L$ using advanced sparse matrix packages ${ }^{12}$ yields eigenfunctions $\psi_{i}$ and eigenvalues $e^{-i \epsilon_{i}}$, where $i=1,2, \ldots, 4 L^{2}$. In Fig. 1 we display the DOS for different system sizes $L$. It is seen that after a proper rescaling all data collapse onto a single curve, as expected at criticality. At $\epsilon \gg \delta$ (where $\delta \sim L^{-7 / 4}$ is the level spacing at $\epsilon=0)$ the DOS scales as $\rho(\epsilon) \sim \epsilon^{1 / 7}$, in agreement with the analytical prediction. ${ }^{4}$ On the other hand, at $\epsilon \sim \delta$ one observes an oscillatory structure qualitatively analogous to the behavior found in the random matrix theory for class $\mathrm{C}{ }^{1}$ 


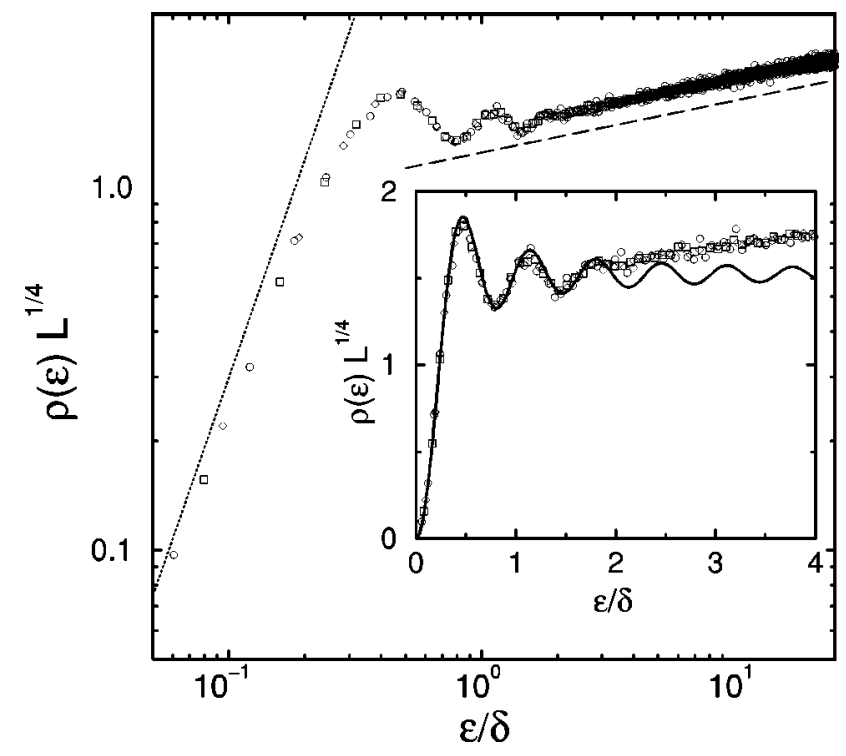

FIG. 1. Scaling plot of the density of states for system sizes $L$ $=16(\diamond), 32(\square)$, and $96(\bigcirc)$. Dashed and dotted lines indicate power laws (dashed: $\epsilon^{1 / 7}$, dotted: $\epsilon^{2}$ ), and $\delta=1 / 2 \pi L^{7 / 4}$ denotes the level spacing. Inset: same data on a linear scale and the result from random matrix theory (Ref. 1) (solid curve).

We now concentrate on the statistics of eigenfunctions with the smallest energies, $|\epsilon| \sim \delta$, for which the correlation length $\xi_{\epsilon} \sim \epsilon^{-4 / 7}$ (Ref. 4) is of the order of the system size. In Fig. 2 (inset) we plot the eigenfunction autocorrelation function [Eq. (1)]. The result implies a power-law behavior $\propto r^{-\eta}$ at distances $1 \ll r \ll L$, with an index $\eta$ close to $1 / 4$. We also show the correlation function of two eigenstates neighboring in energy [the second one in Eq. (2)]. It exhibits a scaling behavior consistent with the above value of $\eta$ but with considerably stronger finite-size effects. On the other hand, our numerics confirms the value $1 / 2$ (Ref. 4) of the exponent governing the decay of $\Pi$ (Fig. 2, main panel). To understand the difference between the two exponents, we turn now to an analytical approach.

Consider a correlation function of two wave functions,

$$
\begin{aligned}
\mathcal{D}\left(e^{\prime}, e ; \epsilon_{1}, \epsilon_{2}\right)= & \left\langle\sum_{i j \alpha \beta} \psi_{i \alpha}^{*}(e) \psi_{j \alpha}(e) \psi_{i \beta}\left(e^{\prime}\right) \psi_{j \beta}^{*}\left(e^{\prime}\right)\right. \\
& \left.\times \delta\left(\epsilon_{1}-\epsilon_{i}\right) \delta\left(\epsilon_{2}-\epsilon_{j}\right)\right\rangle,
\end{aligned}
$$

where $e$ and $e^{\prime}$ are two different edges of the network and $\alpha, \beta=1,2$ are the $\mathrm{SU}(2)$ indices. Introducing the Green function $G\left(e^{\prime}, e ; z\right)=\left\langle e^{\prime}\left|(1-z \mathcal{U})^{-1}\right| e\right\rangle$, we express Eq. (5) as

$$
\begin{aligned}
\mathcal{D}\left(e^{\prime}, e ; \epsilon_{1}, \epsilon_{2}\right)= & (2 \pi)^{-2}\left\langle\operatorname { T r } \left[ G_{R}\left(e^{\prime}, e ; e^{i \epsilon_{1}}\right)\right.\right. \\
& \left.-G_{A}\left(e^{\prime}, e ; e^{i \epsilon_{1}}\right)\right]\left[G_{R}\left(e, e^{\prime} ; e^{i \epsilon_{2}}\right)\right. \\
& \left.\left.-G_{A}\left(e, e^{\prime} ; e^{i \epsilon_{2}}\right)\right]\right\rangle,
\end{aligned}
$$

where $G_{R, A}$ are retarded and advanced Green functions, $G_{R, A}\left(e^{\prime}, e ; e^{i \epsilon_{1}}\right)=G\left(e^{\prime}, e ; e^{i\left(\epsilon_{1} \pm i 0\right)}\right)$. We will calculate Eq. (6) at zero energy, $\epsilon_{1,2} \rightarrow 0$, but finite level broadening,

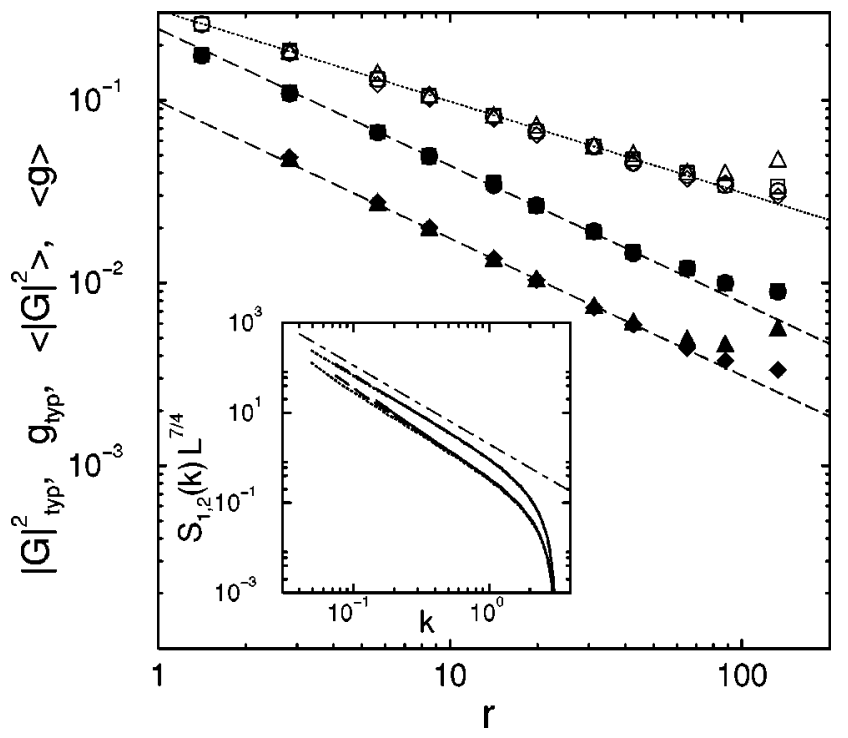

FIG. 2. Scaling of the two-point conductance with a distance $r$ between the contacts: average value (empty symbols), $\langle g\rangle$, and typical value (filled symbols), $g_{\text {typ }}=\exp \langle\ln g\rangle$, in systems of sizes $L=128(\square)$ and $L=196(\bigcirc)$. Also shown is scaling of the twopoint Green function, $\left\langle|G|^{2}\right\rangle$ and $|G|_{\text {typ }}^{2}=\exp \left\langle\ln |G|^{2}\right\rangle \quad(L$ $=128(\triangle), L=196(\diamond))$. The lines correspond to the $r^{-1 / 2}$ (dotted) and $r^{-3 / 4}$ (dashed) power laws. Deviations from power-law scaling at large values of $r$ are due to the finite system size. Inset: Fourier transforms of the one- and two-eigenfunction correlation functions, $S_{1}(r)=\left\langle\left|\psi_{i \alpha}^{2}(e) \psi_{i \beta}^{2}\left(e^{\prime}\right)\right|\right\rangle \quad$ (upper curves) and $S_{2}(r)$ $=\left\langle\psi_{i \alpha}^{*}(e) \psi_{j \alpha}(e) \psi_{i \beta}\left(e^{\prime}\right) \psi_{j \beta}^{*}\left(e^{\prime}\right)\right\rangle$ (lower curves) for $\epsilon_{i, j} \sim \delta$ and $L$ $=128$ (solid), 256 (dashed), and 384 (dotted). The dot-dashed line indicates a power law $S(k) \propto k^{-7 / 4}$ corresponding to $S(r) \propto r^{-1 / 4}$.

$\pm i 0 \rightarrow \pm i \gamma$. The scaling behavior of correlation function (5) at $\epsilon_{1}, \epsilon_{2} \sim \epsilon$ can then be obtained by substituting $\epsilon$ for $\gamma$. We thus need to calculate

$$
\begin{aligned}
\mathcal{D}\left(e^{\prime}, e ; \gamma\right)= & (2 \pi)^{-2}\left\langle\operatorname{Tr}\left[G\left(e^{\prime}, e ; z\right)-G\left(e^{\prime}, e ; z^{-1}\right)\right]\right. \\
& \left.\times\left[G\left(e, e^{\prime} ; z\right)-G\left(e, e^{\prime} ; z^{-1}\right)\right]\right\rangle,
\end{aligned}
$$

with a real $z=e^{-\gamma}<1$. To do this, we make use of the mapping to the classical percolation, following the approach of Ref. 5. We give only a brief outline of the calculation here; details will be published elsewhere. ${ }^{13}$ The Green functions in Eq. (7) are represented as sums over paths; the resulting expression is to be averaged over $\mathrm{SU}(2)$ matrices $U_{f}$ associated with all network edges $f$. The crucial point is that for each edge $f$ only paths visiting it 0 or two times are to be taken into account. In Ref. 5 this was proven for the average Green function $\langle\operatorname{Tr} G(e, e ; z)\rangle$. The proof is based on the observation that $\left\langle U_{f}^{q}\right\rangle=c_{q} \cdot \mathbf{1}$, where $c_{q}=0$ for integer $q \neq 0$, \pm 2 . We generalize the statement to products of two Green functions of the type entering Eq. (7) as well as another two-point correlation function,

$$
\begin{aligned}
\widetilde{D}\left(e^{\prime}, e ; \gamma\right)= & (2 \pi)^{-2}\left\langle\operatorname{Tr}\left[G(e, e ; z)-G\left(e, e ; z^{-1}\right)\right]\right. \\
& \left.\times \operatorname{Tr}\left[G\left(e^{\prime}, e^{\prime} ; z\right)-G\left(e^{\prime}, e^{\prime} ; z^{-1}\right)\right]\right\rangle,
\end{aligned}
$$


corresponding to the $\left\langle\left|\psi_{i}^{2}(e) \psi_{j}^{2}\left(e^{\prime}\right)\right|\right\rangle$ correlator, in the following way. Classifying the paths according to the number of times they return to a link $f$, we obtain expressions of the type $\Sigma_{q_{1}, q_{2}=1}^{\infty}\left\langle\operatorname{Tr} U_{f}^{q_{1}} A U_{f}^{q_{2}} B\right\rangle x^{q_{1}+q_{2}}$ with $A, B \in \mathrm{SU}(2)$ and $x \in \mathbb{R}$. Averaging over $U_{f}$ now yields two terms, proportional to $c_{q_{1}+q_{2}}$ and $c_{q_{1}-q_{2}}$, respectively. While the first one is nonzero only for $q_{1}+q_{2}=2$ traversals of the link, as required, the second one seems to spoil the proof. However, summing over $q_{1}$ at fixed $q_{1}+q_{2}$, we find that such terms cancel in view of $\Sigma_{q} c_{q}=c_{-2}+c_{0}+c_{2}=-1 / 2+1+(-1 / 2)$ $=0$. Having established that only paths visiting each link 0 or two times are to be considered, we can express, in analogy with Ref. 5, correlation functions (7) and (8) in terms of sums over paths representing hulls in the percolation problem. In particular, we get for the products of Green functions entering Eq. (7),

$$
\begin{gathered}
\left\langle\operatorname{Tr} G\left(e^{\prime}, e ; z\right) G\left(e, e^{\prime} ; z\right)\right\rangle=\left\langle\operatorname{Tr} G\left(e^{\prime}, e ; z^{-1}\right) G\left(e, e^{\prime} ; z^{-1}\right)\right\rangle \\
=-2 \sum_{N} P\left(e^{\prime}, e ; N\right) z^{2 N}
\end{gathered}
$$

where $P\left(e^{\prime}, e ; N\right)$ and $P_{1}\left(e^{\prime}, e ; N\right)$ are probabilities that the edges $e$ and $e^{\prime}$ belong to the same loop of the length $N$ (respectively with the length $N$ of the part corresponding to the motion from $e$ to $e^{\prime}$ ). At $z=1$ both Eqs. (9) and (10) reduce to -2 times the probability $P\left(e^{\prime}, e\right)$ that $e$ and $e^{\prime}$ belong to the same loop, which is identical (up to the sign) to the expression for the average conductance $\left\langle g\left(e^{\prime}, e\right)\right\rangle$ at $\epsilon$ $=0$ obtained in Ref. 5 .

The fractal dimension of the percolation hulls is $7 / 4,{ }^{14}$ implying $^{15}$ that $P$ and $P_{1}$ scale as

$$
P(r, N), P_{1}(r, N) \sim N^{-8 / 7} r^{-1 / 4}, \quad r \lesssim N^{4 / 7},
$$

and fall off exponentially fast at $r \gg N^{4 / 7}$ ( $r$ is the distance between $e$ and $e^{\prime}$ ). This yields for the correlation functions in Eqs. (9) and (10) (which we abbreviate as $\left\langle G_{R} G_{R}\right\rangle$, $\left.\left\langle G_{A} G_{A}\right\rangle,\left\langle G_{R} G_{A}\right\rangle\right)$,

$$
\begin{gathered}
\left\langle G_{R} G_{R}\right\rangle=\left\langle G_{A} G_{A}\right\rangle \simeq\left\langle G_{R} G_{A}\right\rangle \sim r^{-1 / 2}, \\
r \ll \xi_{\gamma} \equiv \gamma^{-4 / 7},
\end{gathered}
$$

in full agreement with the scaling argument of Ref. 4 and with our numerics. However, when we substitute Eqs. (9) and (10) in Eq. (7), these leading order terms cancel since $\Sigma_{N}\left[P(r, N)-P_{1}(r, N)\right]=0$. The result is nonzero due to the factors $z^{2 N}$ only, implying that relevant $N$ are now $N$ $\sim \gamma^{-1}$, so that $\left\langle\left(G_{R}-G_{A}\right)\left(G_{R}-G_{A}\right)\right\rangle$ scales differently compared to Eq. (12):

$$
\begin{aligned}
\mathcal{D}\left(e^{\prime}, e ; \gamma\right) & =\frac{1}{\pi^{2}} \sum_{N}\left[P(r, N)-P_{1}(r, N)\right]\left(1-e^{-2 N \gamma}\right) \\
& \sim P\left(r, \gamma^{-1}\right) \gamma^{-1} \sim\left(\xi_{\gamma} r\right)^{-1 / 4}, \quad r \lesssim \xi_{\gamma} .
\end{aligned}
$$
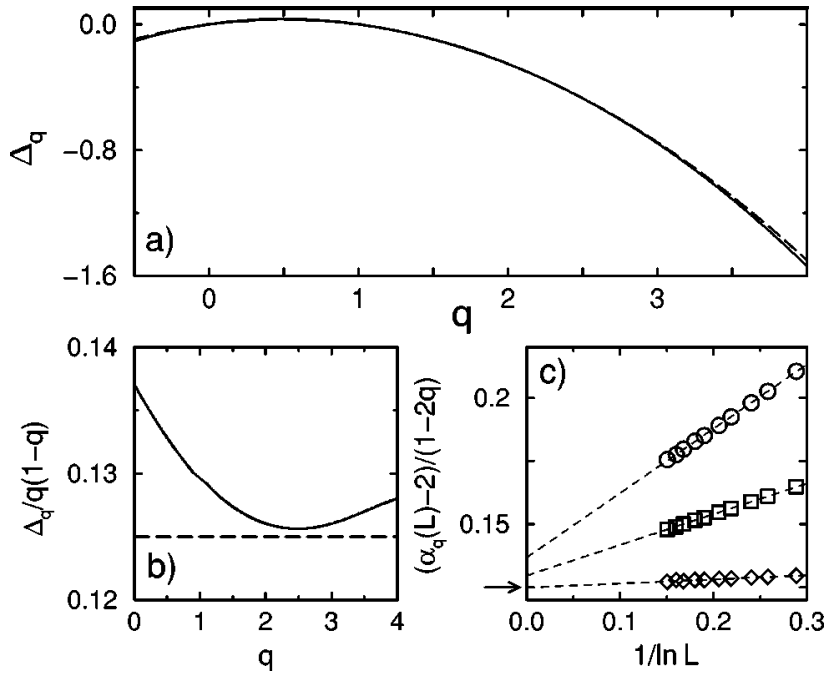

FIG. 3. (a) Anomalous dimension $\Delta_{q}$ extrapolated from ensembles with system sizes $L=16-384$. The dashed line (almost indistinguishable) is the parabola $\Delta_{q}=q(1-q) / 8$. (b) $\Delta_{q} / q(1$ $-q$ ) over $q$ highlighting weak nonparabolicity. (c) Multifractal exponents $\alpha_{q}(L)=-\left\langle|\psi|^{2 q} \ln |\psi|^{2}\right\rangle /\left\langle|\psi|^{2 q}\right\rangle \ln L$ with $q=0$ (○), 1 ( $\left.\square\right)$, and $2(\diamond)$, and extrapolation to an infinite system size (dashed lines): $\quad \alpha_{0}-2=0.137 \pm 0.003, \quad 2-\alpha_{1}=0.130 \pm 0.003$, and (2 $\left.-\alpha_{2}\right) / 3=0.125 \pm 0.001$. The arrow indicates the value $\eta / 2=1 / 8$ of these quantities for a parabolic spectrum.

Now using definition (5) of $\mathcal{D}$ and the DOS scaling, $\rho(\epsilon)$ $\sim \epsilon^{1 / 7} \sim \xi_{\epsilon}^{-1 / 4}$, we find, for $r \lessgtr \xi_{\epsilon}$,

$$
L^{4}\left\langle\psi_{i \alpha}^{*}(e) \psi_{j \alpha}(e) \psi_{i \beta}\left(e^{\prime}\right) \psi_{j \beta}^{*}\left(e^{\prime}\right)\right\rangle \sim\left(r / \xi_{\epsilon}\right)^{-1 / 4} .
$$

The same scaling behavior is obtained for the correlation function $\left\langle\left|\psi_{i \alpha}^{2}(e) \psi_{j \beta}^{2}\left(e^{\prime}\right)\right|\right\rangle .{ }^{13}$ We thus conclude that $\eta$ $=1 / 4$, consistent with our above numerical results. To shed more light on the difference in scaling between $\langle G G\rangle$ (or $\langle g\rangle)$ [Eq. (12)], and $\mathcal{D}$ [Eq. (13)], it is instructive to reverse the logic and to ask how Eq. (12) can be obtained from wave-function correlations (13) and (14). It is straightforward to express $\langle G G\rangle$ through $\mathcal{D}$ in the form of an integral over $\epsilon_{1,2}$ with corresponding energy denominators (dispersion relation). The integral is then dominated by $\epsilon_{1,2}$ $\sim \epsilon(r)$, where $\epsilon(r)$ is defined by $\xi_{\epsilon(r)} \sim r$ (i.e. $\epsilon(r)$ $\sim r^{-7 / 4}$ ). This yields $\langle G G\rangle \sim \mathcal{D}[r ; \epsilon(r)] \sim r^{-1 / 2}$ [we used Eq. (13) in the last step], in agreement with Eq. (12). Therefore, $\langle G G\rangle$ (or $\langle g\rangle$ ) is determined by wave functions with energies $\epsilon(r)$, which transforms $\xi_{\epsilon}^{-1 / 4}$ in Eq. (13) into an additional factor $r^{-1 / 4}$. We will come back to this argument below to obtain an analogous relation for higher moments.

In order to study the whole multifractal spectrum $\Delta_{q}$, we return to numerical simulations. Our procedure based on the evaluation of ensemble averaged moments $\left\langle\left|\psi^{2 q}\right|\right\rangle$ and extrapolation to $L \rightarrow \infty$ was described in detail in Ref. 10. The results for $\tau_{q}$ are shown in Fig. 3(a). The obtained spectrum is parabolic with a high accuracy. A parabolic spectrum is uniquely determined by $\eta, \Delta_{q}=\eta q(1-q) / 2$; the above result $\eta=1 / 4$ thus implies

$$
\Delta_{q} \simeq q(1-q) / 8 .
$$


However, we find clear deviations from the parabolic law [Eq. (15)], as shown in Fig. 3(b). One could ask whether these are not an artifact of uncontrollable finite-size corrections to scaling. We observe, however, an almost perfect scaling for all the moments, yielding, in particular, $\eta$ $=0.252 \pm 0.002$, in a very good agreement with the exact value $\eta=1 / 4$. On the other hand, we find (in standard notations, $\left.\quad \alpha_{q}=d \tau_{q} / d q\right) \quad \alpha_{0}-2=0.137 \pm 0.003 \quad$ and $2-\alpha_{1}$ $=0.130 \pm 0.003$ [Fig. 3(c)], while both these quantities would be equal to $\eta / 2$, should the parabolic law [Eq. (15)] be exact. This makes us believe that the spectrum is only approximately parabolic, in contrast to exact parabolicity found for the QH transition. ${ }^{10}$

Finally, we turn to the statistics of two-point conductances. Generalizing the above argument, we obtain

$$
\begin{aligned}
& \left\langle\left[\operatorname{Tr} G\left(e^{\prime}, e\right) G\left(e, e^{\prime}\right)\right]^{q}\right\rangle \\
& \left.\quad \sim \rho^{2 q}(\epsilon(r)) L^{4 q}\left\langle\left|\psi_{\epsilon_{1}}^{2 q}(e) \psi_{\epsilon_{2}}^{2 q}\left(e^{\prime}\right)\right|\right\rangle\right|_{\epsilon_{1,2} \sim \epsilon(r)} \sim r^{-X},
\end{aligned}
$$

with an index $X_{q}$ related to $\Delta_{q}$ and to the scaling dimension $x_{\rho}$ of the DOS (defined by $\rho(\epsilon) \sim \xi_{\epsilon}^{-x_{\rho}}$ ) as follows (see also Ref. 16);

$$
X_{q}=2 q x_{\rho}+2 \Delta_{q} \text {. }
$$

Using $x_{\rho}=1 / 4$ and Eq. (15) for $\Delta_{q}$, we find

$$
X_{q} \simeq q(3-q) / 4 \text {. }
$$

The same scaling is expected to hold for the two-point conductance, $\left\langle g^{q}\right\rangle \sim r^{-X_{q}}$. However, since $g$ is bounded from above, $g \leqslant 2$, the exponent for $\left\langle g^{q}\right\rangle$ should be a nondecreas- ing function of $q$. Therefore, Eq. (17) will hold only for the moments $\left\langle g^{q}\right\rangle$ with $q \leqslant q_{c}$, where $q_{c} \simeq 3 / 2$ is the maximum of Eq. (17), while for higher $q$ the exponent saturates, $X_{q \geqslant q_{c}}=X_{q_{c}} \simeq 9 / 16$ (these moments are determined by the probability to find $g \sim 1$ ). Equation (17) corresponds to a normal distribution of $\ln g$ (at $r \gg 1$ ) with the average $\langle\ln g(r)\rangle \simeq-\frac{3}{4} \ln r$ and the variance $\operatorname{var}[\ln g(r)] \simeq \frac{1}{2} \ln r$. These arguments are fully confirmed by the results of the numerical simulations shown in Fig. 2.

It remains an open question whether the multifractal exponents $\Delta_{q}$ and $X_{q}$ can be computed by the conformal field theory methods. ${ }^{8,11,16-19}$ Note that our results do not confirm the proposal of Ref. 16, where the value $\eta=1 / 2$ was obtained. Apparently, this indicates that the theory considered in Ref. 16 and obtained ${ }^{18}$ from a particular network model with fine-tuned couplings, does not belong to the SQH universality class.

To summarize, we have studied, by combining numerical and analytical methods, the wave function statistics at the SQH transition. In particular, we have shown, using a mapping to classical percolation, that the index $\eta \equiv-\Delta_{2}$ [defined by Eq. (1)] is equal to $1 / 4$, at variance with the $r^{-1 / 2}$ scaling of the diffusion propagator $\Pi=\left\langle G_{R} G_{A}\right\rangle$. The multifractal spectra of wave functions $\left(\Delta_{q}\right)$ and two-point conductances $\left(X_{q}\right)$ are given with a good accuracy by Eqs. (15) and (17), but show detectable deviations from parabolicity.

Discussions and correspondence with J.T. Chalker, I. Gruzberg, A. LeClair, and J.E. Moore are gratefully acknowledged. This work was supported by the SFB 195 and the Schwerpunktprogramm "Quanten-Hall-Systeme" der Deutschen Forschungsgemeinschaft.
*Also at Petersburg Nuclear Physics Institute, 188350 St. Petersburg, Russia.

${ }^{1}$ A. Altland and M.R. Zirnbauer, Phys. Rev. B 55, 1142 (1997)

${ }^{2}$ V. Kagalovsky, B. Horovitz, Y. Avishai, and J.T. Chalker, Phys. Rev. Lett. 82, 3516 (1999).

${ }^{3}$ T. Senthil, J.B. Marston, and M.P.A. Fisher, Phys. Rev. B 60, 4245 (1999).

${ }^{4}$ I.A. Gruzberg, A.W.W. Ludwig, and N. Read, Phys. Rev. Lett. 82, 4524 (1999).

${ }^{5}$ E.J. Beamond, J. Cardy, and J.T. Chalker, Phys. Rev. B 65, 214301 (2002).

${ }^{6}$ B. Huckestein, Rev. Mod. Phys. 67, 357 (1995); M. Janssen, Phys. Rep. 295, 1 (1998); A.D. Mirlin, ibid. 326, 259 (2000).

${ }^{7}$ M. Janssen, M. Metzler, and M.R. Zirnbauer, Phys. Rev. B 59, 15836 (1999).

${ }^{8}$ M.R. Zirnbauer, hep-th/9905054v2 (unpublished).

${ }^{9}$ R. Klesse and M.R. Zirnbauer, Phys. Rev. Lett. 86, 2094 (2001).
${ }^{10}$ F. Evers, A. Mildenberger, and A.D. Mirlin, Phys. Rev. B 64, 241303(R) (2001).

${ }^{11}$ M.J. Bhaseen et al., Nucl. Phys. B 580, 688 (2000); A.M. Tsvelik, cond-mat/0112008 (unpublished).

${ }^{12}$ J.W. Demmel et al., SIAM J. Matrix Anal. Appl. 20, 720 (1999); R. B. Lehoucq, D. Sorensen, and C. Yang, ARPACK Users guide (SIAM, Philadelphia, 1998).

${ }^{13}$ A.D. Mirlin, F. Evers, and A. Mildenberger, cond-mat/0208451 (unpublished).

${ }^{14}$ H. Saleur and B. Duplantier, Phys. Rev. Lett. 58, 2325 (1987).

${ }^{15}$ For the most recent discussion, see J.E. Moore, Phys. Rev. B 65, 241309 (2002).

${ }^{16}$ D. Bernard and A. LeClair, Nucl. Phys. B 628, 442 (2002).

${ }^{17}$ N. Read and H. Saleur, Nucl. Phys. B 613, 409 (2001).

${ }^{18}$ D. Bernard and A. LeClair, Phys. Rev. B 64, 045306 (2001).

${ }^{19}$ P. Fendley, cond-mat/0006360 (unpublished). 